\title{
COMPARAÇÃo INTERLABORATORIAL PARA ANÁLISE DE DUREZA TOTAL E CLORETO EM ÁGUA
}

\author{
Débora França de Andrade* \\ Escola de Química, Universidade Federal do Rio de Janeiro, Centro de Tecnologia, Bl. E, Ilha do Fundão, 21949-900 \\ Rio de Janeiro - RJ, Brasil \\ Armando Augusto Clemente e Vera Harcar \\ Rede de Tecnologia do Rio de Janeiro, Av. Rio Branco, 125, 200040-006 Rio de Janeiro - RJ, Brasil \\ Jefferson Lee Lima de Melo, Sônia Elisa Carvalho Pereira, Lucia Helena Menezes Santos e Viridiana Santana Ferreira-Leitão \\ Instituto Nacional de Tecnologia, Av. Venezuela, 82, 20081-312 Rio de Janeiro - RJ, Brasil
}

Recebido em 26/8/09; aceito em 29/3/10; publicado na web em 29/6/10

\begin{abstract}
INTERLABORATORIAL COMPARISON FOR ANALYSIS OF TOTAL HARDNESS AND CHLORIDE IN WATER. Interlaboratorial comparison of the determination of hardness and chloride in water had been performed by 38 and 37 laboratories, respectively. In all cases the participating laboratories used its routine methods. Homogeneity and stability testing were performed on the samples sent to the laboratories. The codified results are graphically reported and compared to assigned value, determined by the consensus of the laboratories. Satisfactory results were obtained for 71 and $73 \%$ of the laboratories, considering hardness and chloride determination, respectively.
\end{abstract}

Keywords: interlaboratorial comparison; hardness; chloride.

\section{INTRODUÇÃO}

O ensaio de proficiência laboratorial (EP) apresenta-se como uma valiosa ferramenta para que os laboratórios demonstrem sua competência e busquem o reconhecimento mútuo, adequando assim sua infraestrutura tecnológica aos padrões internacionais, superando desta forma as barreiras técnicas e aumentando sua inserção no comércio exterior. ${ }^{1-5}$

Um dos objetivos das comparações interlaboratoriais é avaliar continuamente a competência técnica dos laboratórios participantes, através da comparação dos resultados obtidos, de modo a oferecer subsídio para a implantação de ações preventivas para melhoria dos procedimentos do laboratório.

Vários são os benefícios para um laboratório advindos da participação em $\mathrm{EP}^{6}$ como, por exemplo, contar com uma avaliação externa, regular e independente da qualidade de seus resultados; ter do organizador do programa uma fonte de assessoramento técnico e orientação sobre problemas analíticos, além de ser capaz de comparar regularmente o seu desempenho com o de outros laboratórios semelhantes, podendo alguns estudos fornecer informações sobre as características de desempenho dos métodos analíticos. Com a crescente demanda por análises químicas e a necessidade da aplicação rigorosa da metrologia, o EP surge como uma ferramenta fundamental para a avaliação da qualidade dos resultados, da capacidade e confiabilidade metrológica dos laboratórios brasileiros. ${ }^{7}$

O EP é uma ferramenta prevista na norma NBR ISO/IEC $17025^{8}$ e deve ser utilizado pelos laboratórios de ensaio para verificação da qualidade dos resultados obtidos. Por causa da necessidade contínua de demonstrar competência para os organismos regulatórios e clientes, os laboratórios vêm, cada vez mais, adotando estas práticas. Dentre as várias técnicas estatísticas empregadas para a avaliação de resultados em programas interlaboratoriais, pode-se destacar a técnica do $Z$-score ${ }^{9-13} \mathrm{O}$ conceito da estatística " $\mathrm{z}$ " da distribuição normal é a base matemática desta técnica.

A necessidade de analisar água sob o ponto de vista físico-químico tem suscitado o interesse da comunidade científica para esta temática

\footnotetext{
*e-mail: debora.franca.andrade@gmail.com
}

e proporcionado o desenvolvimento de novas técnicas analíticas. ${ }^{14} \mathrm{Os}$ métodos normalmente empregados na análise de água são os eletroanalíticos, os espectroscópicos e os cromatográficos, sendo a escolha dependente do parâmetro que se quer medir, do rigor pretendido e dos recursos disponíveis. ${ }^{15-18}$

Devido à necessidade dos laboratórios públicos e privados de análises físico-químicas e microbiológicas em: monitorar os processos de tratamento de águas e esgotos e a qualidade dos efluentes das estações de tratamento lançados em corpos receptores; monitorar a rede de abastecimento público de águas e, fiscalizar as características dos efluentes industriais, faz-se necessária a oferta de programas de ensaio de proficiência (PEP) acessíveis, de modo a garantir a confiabilidade dos resultados analíticos fornecidos por estes. Dentre os vários programas nacionais de EP, somente cinco apresentam em seu escopo parâmetros físico-químicos e microbiológicos de controle de qualidade de águas, esgotos e áreas afins. Os organizadores desses PEP foram a Associação Brasileira de Engenharia Sanitária e Ambiental (ABES), a Associação Rede de Metrologia e Ensaios do Rio Grande do Sul, o Programa de Ensaios de Proficiência para Laboratórios de Águas, Esgotos e Áreas Afins da Companhia Estadual de Águas e Esgotos (PEP-CEDAE), a Rede Metrológica do Rio Grande do Sul e o Centro de Tecnologia Industrial Pedro Ribeiro (SENAI-CETIND).

O PEP-CEDAE, criado em 2004, vem sendo gerenciado e executado pela Divisão de Pesquisas da CEDAE, no Laboratório de Pesquisa e Controle de Qualidade, com apoio do Laboratório de Estudos de Quimiometria do Instituto de Química da Universidade Federal do Rio de Janeiro. Dentre os parâmetros oferecidos, pode-se destacar: pH faixa ácida e alcalina, resíduos não filtráveis totais, resíduos não filtráveis fixos, resíduos não filtráveis voláteis, demanda química de oxigênio, nitrogênio amoniacal, fósforo total, cloretos e coliformes.

Em 2007, a Rede de Tecnologia do Rio de Janeiro/Rede de Laboratórios do Rio de Janeiro, em parceria com o Instituto de Radioproteção e Dosimetria (IRD) e o Instituto Nacional de Tecnologia promoveram o Programa Interlaboratorial em Análise de Água (Quali$\mathrm{H}_{2} \mathrm{O}$ ). Este programa foi financiado pela Financiadora de Estudos e Projetos (FINEP). Os laboratórios participantes não tiveram custos de inscrição e participação, pois o programa foi oferecido visando 
à disseminação da cultura da qualidade nos laboratórios do Brasil. Este projeto teve como objetivo principal prover programas interlaboratoriais em análise de água, com abrangência nacional. Dentre os objetivos específicos do programa, pode-se destacar: avaliar o desempenho individual de laboratórios para os ensaios propostos; prover subsídios aos laboratórios para a identificação e solução de problemas analíticos; agregar valor ao controle da qualidade dos laboratórios; identificar diferenças interlaboratoriais e, contribuir para o aumento da confiabilidade nas medições dos laboratórios.

O Quali- $\mathrm{H}_{2} 0$ ofereceu 10 parâmetros para análise da qualidade da água (potencial hidrogeniônico - $\mathrm{pH}$, alcalinidade, condutividade, turbidez, dureza total, cloreto, colimetria total e $E$. coli. e radioatividade $\alpha$ e $\beta$ total). Dentre os parâmetros oferecidos, no presente trabalho apenas a avaliação do desempenho dos laboratórios participantes para as determinações de dureza total e cloreto será apresentada. Estes parâmetros foram selecionados por apresentarem o maior percentual de laboratórios com desempenho insatisfatório (24\% para dureza total e $13 \%$ para cloreto).

\section{PARTE EXPERIMENTAL}

\section{Cadastro e registro dos laboratórios participantes}

Os laboratórios cadastrados na home-page do evento foram inscritos no programa interlaboratorial, com todas as informações relativas ao desempenho preservadas através de um código numérico de identificação.

\section{Preparo dos itens de ensaio}

Uma solução aquosa de $\mathrm{CaCl}_{2}$ na concentração de $299 \mathrm{mg} \mathrm{L}^{-1}$ foi empregada para determinação de dureza total. Após 1 h de agitação mecânica, 100 frascos, contendo aproximadamente $180 \mathrm{~mL}$ do material de ensaio, foram enviados aos laboratórios. O material de ensaio empregado para a determinação de cloreto foi uma solução aquosa de $\mathrm{NaCl}$ na concentração de $247 \mathrm{mg} \mathrm{L}^{-1}$. Após 1 h de agitação mecânica, 100 frascos de polietileno, contendo aproximadamente $90 \mathrm{~mL}$ do material de ensaio, foram enviados aos laboratórios. O número total de frascos envasados e identificados para cada análise foi calculado levando-se em consideração o número de laboratórios participantes, o teste de homogeneidade, o teste de estabilidade e para suprir a demanda causada por possíveis danos ao material durante o transporte.

\section{Envio dos itens de ensaio}

Os ensaios de dureza total e cloreto foram codificados, respectivamente, como F02/R01/07 e F03/R01/07. Todos os itens de ensaio foram envasados, identificados e enviados por correio. A embalagem e o método de transporte foram escolhidos de modo a preservar as características dos mesmos. Os laboratórios foram orientados a realizar uma inspeção no ato do recebimento dos itens de ensaio, de modo a verificar algum dano que pudesse invalidar os resultados das medições.

\section{Teste de homogeneidade}

Dentre os 100 frascos envasados para análise de dureza total, 9 foram aleatoriamente separados e analisados em duplicata. As análises foram realizadas segundo as instruções enviadas aos laboratórios participantes, utilizando-se o método titrimétrico. ${ }^{15}$ Dentre os 100 frascos inicialmente envasados para determinação de cloreto, 11 foram aleatoriamente separados e analisados em duplicata. As análises foram realizadas segundo as instruções enviadas aos laboratórios participantes, empregando o método argentométrico. ${ }^{19} \mathrm{O}$ teste de homogeneidade foi realizado segundo a ISO Guide $35,{ }^{20}$ entretanto, o protocolo internacional harmonizado ${ }^{21}$ também foi utilizado de modo a disponibilizar uma análise crítica dos resultados obtidos. Para tal, após a determinação quantitativa do analito, utilizou-se a análise de variância fator único (one-way ANOVA $)^{22,23}$ para estimar a variância amostral (entre amostras) e analítica (intra-amostras-duplicatas) e comparou-se a estimativa do desvio padrão amostral com o desvio padrão do método analítico utilizado.

\section{Teste de estabilidade}

Para o teste de estabilidade foram analisados outros 6 itens de ensaio, também separados aleatoriamente dentre os 100 frascos envasados. Estes foram armazenados na temperatura ambiente e analisados, nos tempos 1, 7, 14, 21, 28 e 35 dias, isto é, contando-se desde o momento do envio das amostras aos laboratórios participantes até a data limite estipulada para recebimento dos resultados.

O estudo da estabilidade visa identificar se existe grau de instabilidade nas determinações de dureza total e cloreto ao longo do tempo. A avaliação foi realizada utilizando-se a análise de variâncias em conjunto com a análise de resíduos. É importante ressaltar que a estabilidade das amostras pode ser avaliada através de estudos de curta e de longa duração. Entretanto, neste programa interlaboratorial a estabilidade dos itens de ensaio foi avaliada somente através de estudos de curta duração.

\section{Valor designado}

O valor designado é definido como um valor atribuído a uma grandeza específica e aceito, às vezes por convenção, como tendo uma incerteza apropriada para uma dada finalidade. ${ }^{24}$ Utilizou-se como referência um valor designado para cada parâmetro, obtido através da mediana (md) de cada grupo de dados. A md de um conjunto de valores, ordenados em ordem crescente de grandeza, é o valor situado de tal forma no conjunto que o separa em dois subconjuntos de mesmo número de elementos. ${ }^{25,26}$ Entretanto, se o conjunto de valores tiver um número par de termos, a md será, por definição, a média aritmética dos dois números que ocuparem as posições centrais do conjunto. ${ }^{25,26}$

\section{Métodos de ensaio}

Os laboratórios participantes foram orientados a utilizar seus procedimentos de rotina para a análise dos itens de ensaio. A metodologia empregada para determinação de dureza total baseia-se na detecção titulométrica, onde o quelante etilenodiaminotetracético sal dissódico (EDTA) forma um complexo quando em contato com certos metais. Quando uma pequena quantidade de indicador preto de eriocromo $\mathrm{T}$ é adicionado à solução aquosa contendo íons cálcio e magnésio, na faixa de pH 10, a solução fica com uma cor rósea. Com a adição de EDTA, os íons cálcio e magnésio são complexados, e a solução fica com uma coloração azul, quando o ponto final da titulação é atingido. ${ }^{19}$

Como metodologia alternativa ao método titulométrico clássico, surgiu a técnica multielementar de análise por espectroscopia de emissão atômica com plasma acoplado indutivamente (ICP-OES). ${ }^{27,28}$ O método baseado na ICP-OES apresenta como vantagens: maior precisão, exatidão, sensibilidade e rapidez, quando comparado à titulometria. Entretanto, uma limitação à aplicação desta metodologia é o alto custo do equipamento empregado e a necessidade de corpo técnico mais especializado.

O cloreto pode ser determinado por métodos baseados na gravimetria, na titulometria (argentometria), na potenciometria e em métodos ópticos de análise. Os métodos argentométricos, como o método de 
Mohr e o de Volhard, são extensivamente utilizados em determinação de íons cloreto. ${ }^{29}$ No método de Mohr a concentração dos íons cloreto é determinada diretamente, enquanto que no método de Volhard a detecção do ponto final é feita por meio de titulação reversa. ${ }^{30,31}$

\section{Amplitude interquartílica normalizada}

A amplitude interquartílica normalizada (IQN), medida de dispersão equivalente ao desvio padrão da estatística clássica, foi calculada através da Equação 1.

$$
\mathrm{IQN}=0,74\left(\mathrm{Q}_{3}-\mathrm{Q}_{1}\right)
$$

onde: $\mathrm{Q}_{1}$ (primeiro quartil)é o valor que está situado de tal modo no conjunto de dados, que uma quarta parte $(25 \%)$ dos dados é menor e as três quartas partes restantes (75\%), maiores do que ele; $\mathrm{Q}_{3}$ (terceiro quartil) é o valor que está situado de tal modo no conjunto de dados, que as três quartas partes (75\%) dos dados são menores e uma quarta parte $(25 \%)$, maior que ele; e 0,74 é o fator para uma distribuição normal/Gaussiana.

\section{Coeficiente de variação}

Para expressar a relação percentual da IQN com a md dos valores obtidos utilizou-se o coeficiente de variação (CV), Equação 2. Foi utilizado o conceito da estatística robusta, ${ }^{32}$ onde o $\mathrm{CV}$ é um indicador importante para a análise da variabilidade dos dados.

$$
\mathrm{CV}=(\mathrm{IQN} / \mathrm{md}) * 100
$$

\section{Índice de desempenho}

Os resultados foram avaliados utilizando-se o método do Z-score robusto, onde o desempenho de cada laboratório foi obtido pela comparação da diferença entre seu resultado e o valor designado para o parâmetro (md do grupo), com a medida de dispersão, expressa através do cálculo da IQN. Para cada um dos resultados obtidos pelos laboratórios foi calculado o respectivo Z-score (Z), através da Equação 3:

$$
\mathrm{Z}=(\mathrm{xi}-\mathrm{md}) / \mathrm{IQN}
$$

onde: xi é o resultado obtido pelo laboratório $i$; md é o valor da mediana dos resultados do grupo para o parâmetro e IQN é a amplitude interquartílica normalizada dos resultados do grupo para o parâmetro.

No método do Z-score o desempenho dos laboratórios é classificado como satisfatório, questionável ou insatisfatório, para cada um dos parâmetros, conforme o índice Z (valores em módulo), de acordo com o seguinte critério: ${ }^{1}$ satisfatório quando $|Z| \leq 2$; questionável quando $2<|Z|<3$; e insatisfatório quando $|Z| \geq 3$.

\section{RESULTADOS E DISCUSSÃO}

\section{Abrangência do programa interlaboratorial}

Cinquenta e dois laboratórios pertencentes a treze estados brasileiros participaram deste programa interlaboratorial. A Figura 1 apresenta a distribuição dos laboratórios participantes por estado. A partir dos dados apresentados na Figura 1, observa-se que os estados do Rio de Janeiro (27\%), São Paulo (17\%) e Bahia (13\%) apresentaram o maior percentual de participantes, enquanto os estados do Ceará e Amazonas (2\%) foram os que tiveram o menor percentual de participantes. As regiões sudeste (54\%) e nordeste (29\%) apresentaram maior participação. Já as regiões sul e norte apresentaram, respectivamente, 15 e $2 \%$ de participação. Não houve participação de nenhum laboratório da região centro-oeste. Dentre os laboratórios participantes deste programa interlaboratorial, $28 \%$ foram de instituições públicas e $72 \%$ de empresas privadas. O grande percentual de laboratórios privados envolvidos sugere uma boa perspectiva de mercado para a implantação de PEP em análise de águas e efluentes.

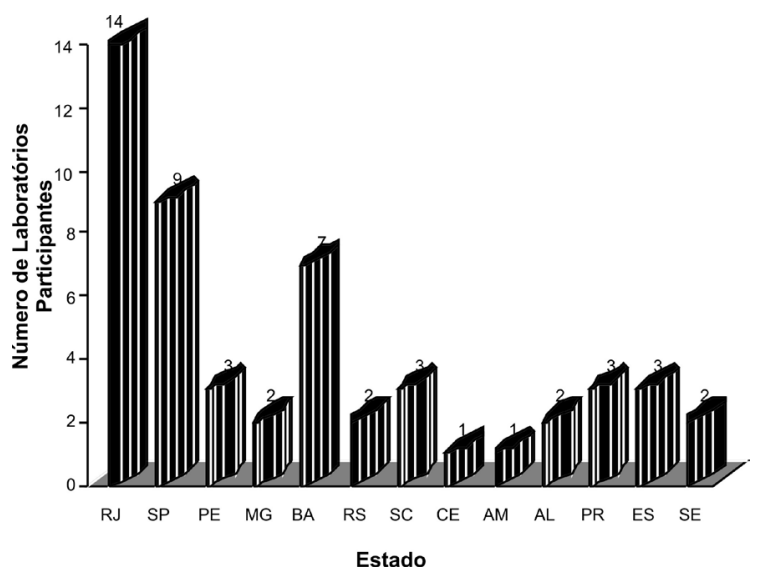

Figura 1. Distribuição dos laboratórios participantes por estado da Federação

\section{Teste de homogeneidade}

Os resultados do teste de homogeneidade obtidos nas determinações de dureza total (F02/R01/07) e cloreto (F03/R01/07) em água são apresentados, respectivamente, nas Tabelas 1 e 2. A partir destes resultados os valores de $\mathrm{F}$ foram calculados segundo a ISO Guide $35,{ }^{20}$ que utiliza a one-way ANOVA, e os dados estão apresentados na Tabela 3.

Tabela 1. Resultados, $\mathrm{mg} \mathrm{L}^{-1} \mathrm{CaCO}_{3}$, obtidos para teste de homogeneidade da análise de dureza total em água (F02/R01/07)

\begin{tabular}{cccccccccc}
\hline \multirow{2}{*}{ Réplica } & 1 & 2 & 3 & 4 & 5 & 6 & 7 & 8 & 9 \\
\hline A & 252 & 252 & 251 & 251 & 250 & 252 & 250 & 252 & 252 \\
B & 252 & 251 & 250 & 252 & 250 & 252 & 250 & 252 & 250 \\
\hline
\end{tabular}

Tabela 2. Resultados, $\mathrm{mg} \mathrm{L}^{-1} \mathrm{Cl}^{\text {, }}$ obtidos para teste de homogeneidade da análise de cloreto em água (F03/R01/07)

\begin{tabular}{|c|c|c|c|c|c|c|c|c|c|c|c|c|}
\hline \multirow{2}{*}{ Fonte de variação } & \multicolumn{2}{|c|}{ Soma Quadrática } & \multicolumn{2}{|c|}{ Graus de Liberdade } & \multicolumn{2}{|c|}{ Média Quadrática } & \multicolumn{2}{|c|}{$\mathrm{F}$} & \multicolumn{2}{|c|}{ Valor-p } & \multicolumn{2}{|c|}{$\mathrm{F}_{\text {crítico }}$} \\
\hline & Dureza & Cloreto & Dureza & Cloreto & Dureza & Cloreto & Dureza & Cloreto & Dureza & Cloreto & Dureza & Cloreto \\
\hline Entre grupos & 0,50 & 0,18 & 1 & 1 & 0,50 & 0,18 & 0,57 & 0,07 & 0,46 & 0,79 & 4,49 & 4,35 \\
\hline Dentro grupos & 14,00 & 50,18 & 16 & 20 & 0,87 & 2,50 & - & - & - & - & - & - \\
\hline Total & 14,50 & 50,36 & 17 & 21 & - & - & - & - & - & - & - & - \\
\hline
\end{tabular}

\begin{tabular}{lccccccccccc}
\hline \multirow{2}{*}{ Réplica } & 1 & 2 & 3 & 4 & 5 & 6 & 7 & 8 & 9 & 10 & 11 \\
\hline A & 145 & 145 & 147 & 143 & 143 & 145 & 143 & 145 & 147 & 143 & 147 \\
B & 145 & 145 & 145 & 143 & 143 & 145 & 143 & 143 & 147 & 145 & 147 \\
\hline
\end{tabular}

Tabela 3. ANOVA de fator único para o teste de homogeneidade da análise de dureza total e cloreto em água 
Através dos dados da Tabela 3, verifica-se mediante aplicação do teste $\mathrm{F}(\alpha-0,05)$, que os valores de $\mathrm{F}_{\text {calculado }}$ para os ensaios de dureza total $(0,57)$ e cloreto $(0,07)$ são menores que os valores de $\mathrm{F}_{\text {crítico }} 4,49$ (dureza total) e de 4,35 (cloreto). Desta forma, concluiu-se que os itens de ensaio utilizados nas determinações de dureza total e cloreto em água podem ser considerados homogêneos.

\section{Teste de estabilidade}

Os resultados do teste de estabilidade obtidos nas determinações de dureza total (F02/R01/07) e cloreto (F03/R01/07) em água são apresentados, respectivamente, nas Tabelas 4 e 5 . A partir destes resultados, as probabilidades de significância (valores-p) foram calculadas, os dados estão apresentados na Tabela 6, onde se observou que os valores de p para os ensaios de dureza total $(0,63)$ e de cloreto $(0,10)$ são maiores que $0,05(95 \%)$. Desta forma, concluiu-se que os itens de ensaio utilizados nas determinações de dureza total e cloreto em água podem ser considerados estáveis. $\mathrm{O}$ valor de $\mathrm{p}$ indica que a regressão é insignificante. Caso o valor de p fosse menor que 0,05 (95\%), tal regressão se apresentaria significativa e o material definido como não estável.

Tabela 4. Resultados, $\mathrm{mg} \mathrm{L}^{-1} \mathrm{CaCO}_{3}$, obtidos para teste de estabilidade da análise de dureza total em água (F02/R01/07)

\begin{tabular}{ccccccc}
\hline \multirow{2}{*}{ Réplica } & \multicolumn{6}{c}{ Semanas } \\
& 1 & 2 & 3 & 4 & 5 & 6 \\
\hline A & 256 & 257 & 254 & 257 & 254 & 259 \\
B & 256 & 257 & 254 & 257 & 254 & 259 \\
\hline
\end{tabular}

Tabela 5. Resultados, $\mathrm{mg} \mathrm{L}^{-1} \mathrm{Cl}^{\text {; }}$, obtidos para teste de estabilidade da análise de cloreto em água (F03/R01/07)

\begin{tabular}{lcccccc}
\hline \multirow{2}{*}{ Réplica } & \multicolumn{6}{c}{ Semanas } \\
& 1 & 2 & 3 & 4 & 5 & 6 \\
\hline A & 142 & 145 & 147 & 147 & 148 & 146 \\
B & 142 & 145 & 147 & 147 & 148 & 146 \\
\hline
\end{tabular}

A estabilidade do material foi determinada através da análise de resíduos dos valores de concentração obtidos ao longo do tempo. Este teste consiste em observar se a regressão linear dos valores de concentração apresenta alguma tendência. Quando a inclinação da reta ou a não linearidade da mesma não forem significativas, o material é considerado estável.

\section{Resultados enviados pelos laboratórios participantes}

A partir dos resultados enviados pelos laboratórios participantes verificou-se que apenas duas técnicas analíticas (titulometria e ICP-OES) foram aplicadas para a determinação de dureza total. A grande maioria dos laboratórios $(87 \%)$ empregou a titulometria no ensaio. Quando a titulometria foi utilizada no ensaio de dureza total, observou-se que $70 \%$ dos laboratórios apresentaram desempenho satisfatório. Entretanto, quando a técnica da ICP-OES foi utilizada, o percentual de laboratórios com desempenho satisfatório subiu para $80 \%$, sugerindo a vantagem da técnica instrumental sobre o procedimento clássico.

Para a determinação de cloreto foram utilizadas três técnicas (titulometria, cromatografia iônica e potenciometria). A grande maioria dos laboratórios (84\%) utilizou a titulometria para as determinações. Embora esta técnica seja considerada menos precisa e rápida em comparação à cromatografia iônica, os resultados mostraram que apenas 13\% dos laboratórios apresentaram desempenho insatisfatório. Quando a titulometria foi empregada na determinação de cloreto, observou-se que $74 \%$ dos laboratórios apresentaram desempenho satisfatório. Contudo, quando a técnica da cromatografia iônica foi utilizada, o percentual de laboratórios com desempenho satisfatório subiu para $80 \%$, confirmando a vantagem da técnica instrumental sobre a clássica. Apenas 3\% dos laboratórios participantes utilizaram a potenciometria. Esta técnica é bastante simples e com bom custo-benefício, e deve ser utilizada com restrições na quantificação de íons, somente quando se pretender estimar ordens de grandeza das concentrações iônicas e nunca para determinações quantitativas.

Como metodologia alternativa aos métodos clássicos (gravimétricos e titulométricos) para determinação de cloreto, surgiu a técnica de cromatografia iônica. Através desta técnica é possível determinar íons com precisão e rapidez e, ao mesmo tempo, separar diferentes espécies químicas do mesmo elemento. A cromatografia iônica é um método mais sofisticado, permitindo maior precisão, exatidão e melhor detectabilidade para os íons cloreto quando comparado com a argentometria. A cromatografia iônica foi empregada por $13 \%$ dos laboratórios participantes.

\section{Mediana, amplitude interquartílica normalizada e coeficiente de variação}

O valor designado para as determinações de dureza total e cloreto foi obtido através da md dos resultados fornecidos pelos laboratórios. A md é normalmente empregada quando há valores extremos que poderiam distorcer o valor da média amostral.

Quando não se dispõe de valor de referência do analito, o mesmo poderá ser estimado através da média dos resultados fornecidos pelos laboratórios, ${ }^{33}$ excluídos os valores dispersos após a aplicação de testes de rejeição como os de Cochran e de Grubbs. ${ }^{34}$ Entretanto, mesmo aplicando os testes para identificação dos valores dispersos, dependendo do número de graus de liberdade (neste caso, número de laboratórios participantes - 1), a estimativa da média poderá ser afetada pelos valores extremos. Desta forma, de modo a minimizar a influência de resultados extremos nas estimativas da média e desvio padrão, optou-se pela utilização de técnicas estatísticas robustas, onde são empregadas a md e a IQN.9,12 No cálculo do CV também foi utilizado o conceito da estatística robusta. Vale lembrar que a IQN na estatística robusta é comparável ao desvio padrão na estatística clássica. A IQN é um índice de variabilidade bastante estável, uma vez que não é influenciada pelos valores extremos e leva em consideração a totalidade dos valores da variável em estudo.

A Tabela 7 apresenta os valores da mediana (md), amplitude interquartílica normalizada (IQN), coeficiente de variação $(C V)$ e número de laboratórios participantes (NLP) para as determinações de dureza total e cloreto, obtidos do conjunto de laboratórios.

Tabela 6. Teste de estabilidade do material de ensaio para a determinação da dureza total e cloreto

\begin{tabular}{lcccccccccc}
\hline & \multicolumn{2}{c}{ Soma Quadrática } & \multicolumn{2}{c}{ Graus de Liberdade } & \multicolumn{2}{c}{ Média Quadrática } & \multicolumn{2}{c}{ F } & \multicolumn{2}{c}{ Valor-p } \\
& Dureza & Cloreto & Dureza & Cloreto & Dureza & Cloreto & Dureza & Cloreto & Dureza & Cloreto \\
\hline Regressão & 1,16 & 12,01 & 1 & 1 & 1,16 & 12,01 & 0,26 & 4,44 & 0,63 & 0,10 \\
Residual & 17,68 & 10,82 & 4 & 4 & 4,42 & 2,70 & - & - & - \\
Total & 18,83 & 22,83 & 5 & 5 & - & - & - & - & - & - \\
\hline
\end{tabular}


Tabela 7. Mediana (md), amplitude interquartílica normalizada (IQN), coeficiente de variação $(\mathrm{CV})$ e número de laboratórios participantes (NLP) para as determinações de dureza total e cloreto

\begin{tabular}{lcccc}
\hline & md & IQN & CV & NLP \\
\hline Dureza Total $\left(\mathrm{mg} \mathrm{L}^{-1} \mathrm{CaCO}_{3}\right)$ & 276,5 & 12,7 & 4,6 & 38 \\
Cloreto $\left(\mathrm{mg} \mathrm{L}^{-1} \mathrm{Cl}^{-}\right)$ & 149,0 & 6,4 & 4,3 & 37 \\
\hline
\end{tabular}

Os resultados apresentados na Tabela 7 demonstram que a IQN para a determinação de dureza total $(12,7)$ é praticamente o dobro da IQN para a determinação de cloreto $(6,4)$. A IQN é expressa na mesma unidade dos dados, desta forma limita o seu emprego quando desejamos comparar duas ou mais séries de valores, relativamente à sua dispersão ou variabilidade, quando expressas em unidades diferentes. Assim, para contornar essas dificuldades e limitações, caracterizou-se a dispersão ou variabilidade dos dados em termos relativos ao seu valor médio, através do $\mathrm{CV}$, também chamado de desvio padrão relativo. Através dos dados apresentados na Tabela 7 observaram-se variabilidades semelhantes entre as determinações de dureza total e cloreto através dos valores de CV.

\section{Índice de desempenho, interpretação dos resultados e representação gráfica}

Os resultados obtidos pelos laboratórios foram estatisticamente avaliados usando a técnica do $Z$-score robusto. $\mathrm{O}$ valor $Z$-score de cada laboratório foi calculado através da Equação 3 e encontra-se representado nas Figuras 2 e 3. A Tabela 8 apresenta o desempenho dos laboratórios em satisfatório, questionável e insatisfatório, para as determinações de dureza total e cloreto.

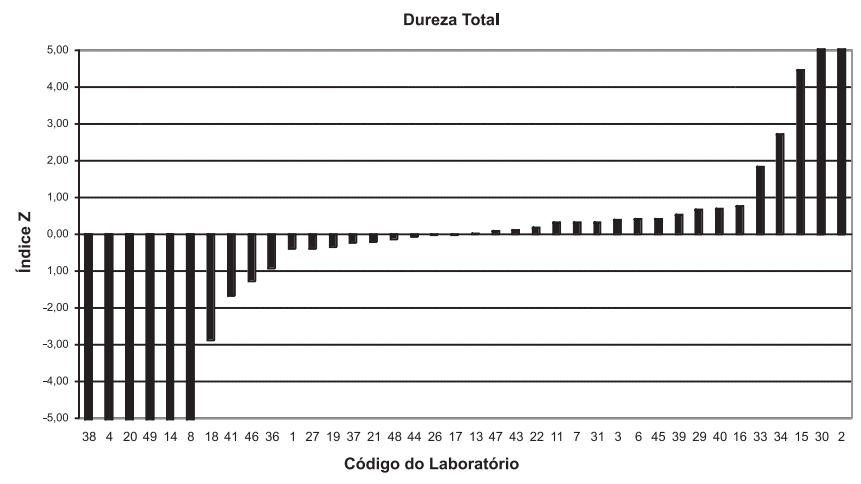

Figura 2. Gráfico do Z-score robusto para a determinação da dureza total

Cloreto

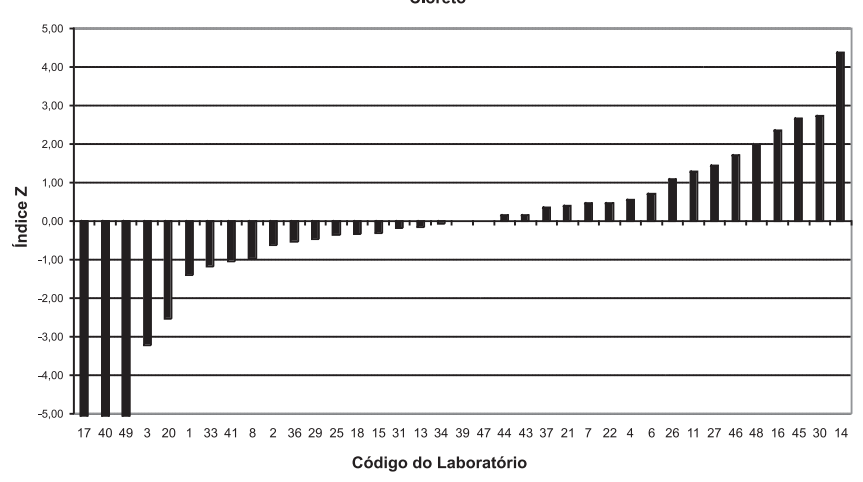

Figura 3. Gráfico do Z-score robusto para a determinação de cloreto

Através dos resultados apresentados na Tabela 8 observou-se que a determinação de dureza total apresentou um percentual de laboratórios com desempenho insatisfatório (24\%), relativamente
Tabela 8. Desempenho dos laboratórios participantes para as determinações de dureza total e cloreto

\begin{tabular}{lccccc}
\hline Dureza Total & & \multicolumn{4}{c}{ Cloreto } \\
\hline Desempenho & NLP & $(\%)$ & Desempenho & NLP & $(\%)$ \\
Satisfatório & 27 & 71 & Satisfatório & 27 & 73 \\
Questionável & 2 & 5 & Questionável & 5 & 14 \\
Insatisfatório & 9 & 24 & Insatisfatório & 5 & 13 \\
\hline
\end{tabular}

NLP - Número de laboratórios participantes

maior em comparação ao desempenho para a determinação de cloreto $(13 \%)$.

\section{CONCLUSÕES}

Através da comparação interlaboratorial, foi possível avaliar os resultados obtidos pelos laboratórios que determinaram a dureza total e o cloreto em amostras de água, utilizando diferentes técnicas analíticas. Observou-se que apenas duas técnicas analíticas (titulometria e ICP-OES) foram utilizadas para a determinação de dureza total. Já para a determinação de cloreto, os laboratórios utilizaram as técnicas da titulometria, cromatografia iônica e potenciometria. É importante ressaltar que a grande maioria dos laboratórios empregou a titulometria nas determinações de dureza total (87\%) e cloreto (84\%). Entretanto, quando foram empregadas técnicas instrumentais em substituição à titulometria, foi observado um aumento do percentual de laboratórios com desempenho satisfatório, sugerindo então a aplicação de técnicas instrumentais para a melhoria da qualidade dos resultados emitidos pelos laboratórios.

Analisando-se os dados reportados pelos laboratórios, pode-se observar que o parâmetro de dureza total apresentou um maior percentual de laboratórios com desempenho insatisfatório (24\%), em comparação ao parâmetro cloreto (13\%). Acredita-se que isto se deva ao fato de para o parâmetro dureza total ter-se empregado em suas determinações um menor percentual de técnicas instrumentais, em comparação ao parâmetro cloreto. No caso de um resultado insatisfatório, espera-se que o laboratório registre não conformidade, identifique as possíveis causas que levaram a ela e aplique as ações corretivas necessárias, com prazos e responsáveis para sua implantação. Cabe salientar que é necessária uma participação constante em programas interlaboratoriais para uma avaliação adequada da confiabilidade dos resultados emitidos pelos laboratórios.

\section{AGRADECIMENTOS}

Aos parceiros do IRD pela condução do programa no que se refere às análises de radioatividade $\alpha$ e $\beta$ total, aos laboratórios participantes e à FINEP pelo apoio financeiro.

\section{REFERÊNCIAS}

1. Associação Brasileira de Normas Técnicas; NBR/ISO/IEC GUIA 43-1 - Ensaios de Proficiência por Comparações Interlaboratoriais. Parte 1: Desenvolvimento e Operação de Programas de Ensaios de Proficiência, Rio de Janeiro, 1999.

2. Associação Brasileira de Normas Técnicas; NBR/ISO/IEC GUIA 43-2, Ensaios de Proficiência por Comparações Interlaboratoriais. Parte 2: Seleção e Uso de Programas de Ensaios de Proficiência por Organismos de Credenciamento de Laboratórios, Rio de Janeiro, 1999.

3. Miller, W. G.; Clin. Biochem. 2009, 42, 232.

4. Ferrero, C.; Casaril, M.; Measurement 2009, 42, 1502.

5. Glavič-Cindro, D.; Korun, M.; Appl. Radiat. Isot. 2009, 67, 683. 
6. Eurachem Netherland, working group on "Interlaboratory Studies"; Statistics and Assessment of Interlaboratory Studies, 1996.

7. Ensaios de Proficiência - Requisitos para Provedores e Banco de Dados, Ed. Interciência: Rio de Janeiro, 2000.

8. Associação Brasileira de Normas Técnicas; NBR/ISO/IEC 17025, Requisitos Técnicos para a Competência Técnica de Laboratório de Ensaios e Calibração, Rio de Janeiro, 2005.

9. Chui, Q. S. H.; Bispo, J. M. A.; Iamashita, C. O.; Quim. Nova 2004, 27, 993.

10. Thompson, M.; Wood, R.; Pure Appl. Chem. 1994, 65, 2123.

11. Firth, J.; Protocol for the Contest Soils Analysis Proficiency Testing Scheme, $2^{\text {nd }}$ ed., Lab. Govern. Chemist: Teddington, 1996.

12. FAPAS - Protocol for the Food Analysis Performance Assessment Scheme; FAPAS Secretariat, CSL, Sand Hutton: York, $5^{\text {th }}$ ed., 1997.

13. Shepherd, J. A.; Blake, G. M.; J. Clin. Densitom. 2007, 10, 349.

14. Simões, M.; Rev. da UNESP 2008, 27, 161.

15. Rodier, J.; L'analyse de l'eau - Eaux naturelles, eaux résiduaires, eau de mer, $8^{a}$ éd., Dunod: Paris, 2005.

16. Gonçalves, M. L. S. S.; Métodos Instrumentais para Análise de Soluções: Análise Quantitativa, $4^{\mathrm{a}}$ ed., Ed. Fundação Calouste Gulbenkian: Lisboa, 2001.

17. Nollet, M. L. L.; Handbook of Water Analysis, $2^{\text {nd }}$ ed., Marcel Dekker: New York, 2000.

18. Tonetto, E. M.; Bonotto, D. M.; Rev. Bras. Geociênc. 1999, 18, 303.

19. Clesceri, L. S.; Greenberg, A. E.; Eaton, A. D.; Standard Methods for the Examination of Water and Wastewater, $20^{\mathrm{th}} \mathrm{ed}$., Apha: Washington, 1998.

20. International Organization for Standardization, ISO Guide 35 (Draft); Certification of Reference Materials - General and Statistical Principles, Geneva, 2003.
21. Thompson, M.; Ellison, S. L. R.; Wood, R.; Pure Appl. Chem. 2006, 78, 145.

22. Todorov, V.; Filzmoser, P.; Comp. Stat. Data Anal. 2010, 54, 37.

23. Li-Wen, X.; Song-Gui, W.; Stat. Probab. Lett. 2008, 78, 963.

24. VIM - Vocabulário Internacional de Termos Fundamentais e Gerais de Metrologia; $3^{\mathrm{a}}$ ed., INMETRO: Rio de Janeiro, 2003.

25. Leite, F.; Validação em Análise Química, $4^{\mathrm{a}}$ ed., Ed. Átomo: Campinas, 2002.

26. Cienfuegos, F.; Estatística Aplicada ao Laboratório, $1^{\text {a }}$ ed., Ed. Interciência: Rio de Janeiro, 2005.

27. Barnhisel, R.; Bertsch, P. M. Em Methods of Soil Analysis. Part 2. Chemical and Microbiological Properties; Page, A. L.; Miller, R. H.; Keeney, D. R., eds; Am. Soc. Agron.: Madison, 1982.

28. Soltanpour, P. N.; Jones Jr., J. B.; Workman, S. M. Em ref. 27.

29. Gaines, T. P.; Parker, M. B.; Gascho, G. J.; Agron. J. 1984, 76, 371.

30. Ohweiler, O. A.; Teoria e Prática da Análise Quantitativa Inorgânica, $1^{a}$ ed., Ed. UnB: Brasília, 1968.

31. Jeffery, G. H.; Basset, J.; Mendham, J.; Denny, R. C.; Vogel - Análise Química Quantitativa, 5 ed., Ed. Guanabara Koogan: Rio de Janeiro, 1992.

32. Daszykowski, M.; Kaczmarek, K.; Heyden, Y. V.; Walczak, B.; Chemom. Intell. Lab. Syst. 2007, 85, 203.

33. Chui, Q. S. H.; Sakuma, A. M.; Okada, I. A.; Maio, D. D.; Olivieri, J. C.; Resumos do II Inter-American Congress on Metrology in Chemistry, São Paulo, Brasil, 2000.

34. International Organization for Standardization, ISO/DIS 5725; Accuracy (trueness and precision) of Measurement Methods and Results. Part 2: A basic method for the determination of repeatability and reproducibility of a standard measurement method, Geneva, 1990. 